\title{
Philosophiques
}

\section{Marx : entre l'idéalisme radical et l'individualisme anarchique}

\section{Ulysses Santamaria et Alain Manville}

Volume 11, numéro 2, octobre 1984

Égalité, justice et différence

URI : https://id.erudit.org/iderudit/203259ar

DOI : https://doi.org/10.7202/203259ar

Aller au sommaire du numéro

Éditeur(s)

Société de philosophie du Québec

ISSN

0316-2923 (imprimé)

1492-1391 (numérique)

Découvrir la revue

\section{Citer cet article}

Santamaria, U. \& Manville, A. (1984). Marx : entre l'idéalisme radical et l'individualisme anarchique. Philosophiques, 11(2), 299-333.

https://doi.org/10.7202/203259ar

\section{Résumé de l'article}

Si Marx aujourd'hui doit retrouver la radicalité qui a porté en ses premiers commencements sa pensée révolutionnaire, il faut rompre définitivement avec les évidences les mieux établies de son interprétation. La pensée de Marx, loin de pouvoir être rattachée au sol du matérialisme, s'enracine au plus profond de l'idéalisme allemand dans ce mouvement de pensée qui vient contester en ses fondements ultimes la métaphysique et son ordre, l'ordre d'un entendement captif de l'horizon borné de la positivité. Pensée de subversion de l'ordre de la positivité, la pensée de Marx se laisse alors définir comme un mouvement de déstabilisation du monde, mise en abyme de son fondement. Cette disqualification an-archique de l'arche est au principe de la pensée de la révolution radicale et de la mise en question fondamentale du pouvoir. Dans l'espace ouvert par cette double Bestimmung, idéaliste, an-archique, la pensée de Marx, en opposition à toute interprétation qui y voit une pensée du social, définit un individualisme radical qui se veut la critique absolue de toute volonté d'édification d'un nouvel ordre, individualisme qui ne laisse plus rien prévaloir qui fasse violence à la valeur de sens de l'autonomie. 


\title{
MARX : ENTRE L'IDÉALISME RADICAL ET L'INDIVIDUALISME ANARCHIQUE
}

\author{
par Ulysses Santamaria et Alain Manville
}

RÉSUMÉ. Si Marx aujourd'hui doit retrouver la radicalité qui a porté en ses premiers commencements sa pensée révolutionnaire, il faut rompre définitivement avec les évidences les mieux établies de son interprétation. La pensée de Marx, loin de pouvoir être rattachée au sol du matérialisme, s'enracine au plus profond de l'idéalisme allemand dans ce mouvement de pensée qui vient contester en ses fondements ultimes la métaphysique et son ordre, l'ordre d'un entendement captif de l'horizon borné de la positivité. Pensée de subversion de l'ordre de la positivité, la pensée de Marx se laisse alors définir comme un mouvement de déstabilisation du monde, mise en abyme de son fondement. Cette disqualification an-archique de l'arché est au principe de la pensée de la révolution radicale et de la mise en question fondamentale du pouvoir. Dans l'espace ouvert par cette double Bestimmung, idéaliste, an-archique, la pensée de Marx, en opposition à toute interprétation qui y voit une pensée du social, définit un individualisme radical qui se veut la critique absolue de toute volonté d'édification d'un nouvel ordre, individualisme qui ne laisse plus rien prévaloir qui fasse violence à la valeur de sens de l'autonomie.

ABSTRACT. The road to Marx's thought supposes as its condition a movement of radical depositioning in relation to the classically established guidelines for journeying through his work. Marx's thought, in opposition to any interpretation which sees therein a reflection on the social sphere, defines a radical form of individualism which is intended as the absolute criticism of any desire to construct a new order, an individualism which allows absolutely nothing which might obstruct the value of the meaning of autonomy to prevail.

\section{MARX ET LE MARXISME}

« Le marxisme n'est pas venu au monde comme un produit authentique de la manière de penser de Karl Marx mais comme un fruit légitime de l'esprit de Friedrich Engels ». Cette affir- 
mation de Maximilien Rubel ne trouve pas meilleure illustration que cette phrase qu'Engels prononça dans son éloge funèbre de Marx au cimetière de High Gate à Londres : "Tout comme Darwin a découvert la loi d'évolution de la nature organique, Marx a découvert la loi d'évolution de l'histoire humaine". Engels poursuit : " . . . son nom et son œuvre vivront à travers les siècles ». Ces quelque mots d'Engels ouvrent une histoire, l'histoire du marxisme, en même temps qu'ils en ferment une autre, l'histoire de la pensée de Marx qui y trouve son point d'exténuation. L'histoire du marxisme, c'est cette histoire qui fait encore de nos jours époque et qui, cent ans après la mort de Marx, continue d'être, de façon dominante, notre histoire. Pour le premier siècle qui a suivi la disparition de Marx, la prédiction d'Engels semble donc s'être vérifiée. Pourtant derrière ce qui peut passer pour une réussite se cache un profond échec. Si l'œuvre de Marx a bien réalisé son devenir-monde, c'est sous la forme d'un paradoxe où l'histoire du marxisme, malgré tous ses succès, présente cependant un véritable anachronisme. Le marxisme, en effet, n'a su s'imposer à l'histoire qu'en lui devenant indifférent. Le triomphe mondain du marxisme a donc été sa perte historique comme puissance critique et foyer d'une mise en question radicale de l'état de chose existant. Marx est devenu le nom référenciel d'un corps doctrinal qui ne définit plus que la répétition du déjà là, doctrine au service de la positivité, incapable d'intervenir de façon signifiante dans le cours du monde qui en a fait l'un de ses facteurs positifs. À l'origine discours contre l'ordre du monde, il tient aujourd'hui le discours du monde. Ce rabaissement de la figure de Marx qui lui a ravi toute pertinence historique, son rapport d'intempestivité au monde, est peut-être à rapporter à une autre forme du paradoxe qui caractérise le marxisme, le fait que Marx comme figure d'une pensée questionnante est le plus souvent radicalement absent dans le marxisme. Nous retrouvons ici le second moment de notre affirmation initiale, la fermeture de la pensée de Marx dans le mouvement par où le marxisme est venu entamer son histoire.

\section{MARX EN 1983 : UN MARX IMAGINAIRE ?}

La pensée de Marx se serait donc perdue dans le mouvement de fondation du marxisme. La pensée de Marx, mais où faut-il la situer ? Si Engels est bien, comme toute une tradition critique 
de l'interprétation de Marx l'a justement reconnu, à l'origine du marxisme, on ne peut simplement exclure, comme certains ont essayé de le faire, Marx de cette fondation. Marx est cofondateur du marxisme et aucune ligne de démarcation tirée entre Marx et Engels ne pourra effacer cette donnée de la pensée de Marx. Le Marx historique, celui qui meurt en 1883, est resté sous la loi énoncée par Hegel selon laquelle chacun est fils de son temps. Le Marx historique, c'est-à-dire le Marx réel n'a pas su rejoindre la radicalité de sa propre pensée, il est resté enfermé dans l'espace théorique de la métaphysique. De ce point de vue, Marx est un théoricien qui appartient de plein droit à l'épistème du XIX ${ }^{\mathrm{e}}$ siècle $^{1}$, au socle d'une pensée orientée de part en part par les valeurs dominantes du positivisme et la visée de la rationalité du discours des sciences. Tous les manifestes et toutes les prises de parti pour la révolution socialiste n'y changent rien. Marx a été, est et reste l'exemple d'un effort pour lier la volonté critique et la volonté de savoir positif ${ }^{2}$. Nietzsche avait raison dans sa critique du socialisme, à cette réserve près que si la pensée historique de Marx appartient bien à ce mouvement d'idées en dernière instance réactionnaire qui réactive les vieilles valeurs morales et sert en définitive le vieil ordre du monde, il reste néanmoins que la pensée de Marx, se pensée épochale, ne se résume ni ne se laisse réduire au devenir réalité qu'elle a connu.

Il y a en Marx encore retenu, caché, l'éclair d'une pensée qui non seulement n'a rien de commun avec la pensée historique

1. Cette appartenance a été remarquée par nombre d'interprètes et elle est une des causes essentielles du non abourissement de la pensée de Marx qui trouve dans une rupture radicale avec la pensée positive la première condition de son déploiement cohérent.

2. C'est à ce couple, qui recouvre en sa signification essentielle un véritable antagonisme, que renvoie la distinction opérée par $\mathbf{M}$. Horkeimer entre théorie traditionnelle et théorie critique. Le savoir positif et la volonté théorique qui en est le ressort est cette forme de l'activité connaissante qui reste nécessairement (pour des raisons, tout d'abord, méthodologique et puis, fondamentalement, pour des raisons ontologiques en raison du sens de l'être qui est au fondement de son mode de pensée) prisonnière de l'horizon limité de la positivité. À la volonté de savoir positif s'oppose un mode de pensée critique commandé par un autre intérêt de la raison, non plus celui d'un savoir immédiatement exploitable, mais celui de la liberté, le Vernunftinteresse der Freineit de Kant. Différence des modes de pensée qui nous renvoie, comme le note Horkheimer, non pas à une différence de l'objet mais à l'attitude du sujet connaissant (cf. M. Horkheimer, Traditionelle und kritische theorie, Fischer Tachenbuch, p. 29). A. Schmidt dans son livre consacré à la théorie critique peut noter à propos de la théorie critique et de son mode de pensée : " Les catégories du mide de pensée critique sont situées historiquement, liées à l'expérience de l'activité et de l'effort propre, à l'existence de la volonté dans le sujet. C'est cela proprement qui fonde la différence entre la théorie traditionnelle et la théorie critique. Elle est tout d'abord moins une différence d'objet que de sujet ". (Die kritische theorie als geschichtsphilosophie, Hanser, p. 84). 
de Marx, mais en est la critique la plus radicale, le procès de déconstruction accompli : pensée originelle qui n'a pas su trouver chez Marx de devenir effectif mais dont l'éclat continue néanmoins d'aimenter l'intérêt de tous ceux qui, cent ans après la mort de Marx et malgré l'existence massive du marxisme, soupçonnent toujours en elle quelque chose qui continue d'interroger notre temps, de lui poser question. Pensée épochale qui seule nous semble justifier la place de Marx auprès de Nietzsche et de Freud comme l'une des trois figures qui marquent la fracture qui ouvre à notre modernité. Pensée épochale, cette pensée en ce qu'elle a de signifiant n'est pas directement lisible dans le texte qui la porte. Elle obéit à cette loi mise en évidence par Hiedegger : " plus grande est l'œuvre d'un penseur et d'autant plus riche est l'impensé que cette œuvre renferme, c'est-à-dire ce qui émerge pour la première fois et grâce à elle, comme n'ayant pas encore été pensé ». Aussi si le mot d'ordre d'un retour au concret de Marx était, il y a vingt ans, lire Marx, il s'énonce aujourd'hui sous l'impératif d'une suspension de la lecture qui ouvre à son Penser. Penser Marx cela signifie alors penser cette pensée dans ce qui fait son éponchalité, c'est-à-dire dans ce qui est pour elle le plus décisif, son impensé, en sachant alors que si Marx fait encore sens pour nous aujourd'hui, ce ne peut être qu'un Marx inventé, un Marx imaginaire qui n'existe pas mais qui est à produire, une fiction à réaliser. Mais pour ce faire, il faut au préalable avoir rompu avec un ensemble de préjugés. Le chemin en direction de la pensée de Marx suppose comme sa condition un mouvement de dépositionnement radical par rapport aux repères classiquement établis pour parcourir l'œuvre de Marx. Il y a nécessité d'une redéfinition complète de toutes les présuppositions qui jusque là ont constitué comme le système des évidences de toute interprétation de Marx. Un tel travail de désimbrication de la pensée de Marx du système des préjugés dominants a été depuis quelques années, en France et à l'étranger, entrepris par quelques interprètes qui, contre l'"establishment" de l'interprétation de Marx ont avancé des thèses aussi originales que fécondes reconnaissant dans la pensée de Marx une profondeur problématique qui lui a redonné ses véritables enjeux ${ }^{3}$. Enjeux d'une pensée problématique complexe

3. On peut citer les travaux de M. Henry (Marx, Paris, Gallimard, 1976), de J.L. Petit (Du travail vivant au système des actions, Paris, Seuil, 1980), de G. Haarsche Marx, Bruxelles, 
où le questionnement ne se limite pas à un domaine d'étant particulier, à une région définie et limitée de l'être, mais dépassant les limites de toute forme d'ontologie régionale, engage l'être dans sa totalité, interroge en direction de l'être et de son sens et comme telle transgresse l'horizon borné qui retient en soi tous les discours et pseudo-discours des sciences. Dimension essentielle de la pensée de Marx qui d'un côté, à être perdue ou ignorée, lui enlève toute pertinence critique et historique et de l'autre a pu conduire certains interprètes à y voir le projet d'une nouvelle métaphysique (métaphysique de la vie chez Michel Henry, métaphysique du travail chez A. Franzki). Cependant si Marx est penseur d'une pensée qui à la portée d'une réflexion métaphysique, métaphysique n'y signifie plus le système de sens qui a dominé depuis Platon la détermination du sens de l'être mais un mouvement d'interrogation meta-ta-physis qui, allant au delà de l'étant, en questionne le sens d'être et, ce faisant, met en question le monde en son fondement même. Qualité de la pensée de Marx qui en fait malgré la dimension métaphysique de la question qui la porte une pensée non métaphysique, pensée qui si elle interpelle bien la totalité de l'étant et la solidité de son fondement n'a plus pour intérêt et comme objectif la fondation de l'être mais à l'inverse la subversion, la destruction de toutes les fondations, de tout ce par quoi la métaphysique s'assure sa domination. Si il y a donc chez Marx quelque chose comme une pensée de l'être, ce n'est plus au sens de Platon et de toute la tradition métaphysique qui l'a suivi mais comme une pensée requise par le projet de la révolution, pensée qui n'est donc plus système d'affirmations qui prétend retrouver et recomposer la vérité de l'être mais questionne l'être en direction de son dépassement ${ }^{4}$.

Édition de l'Université de Bruxelles, 1982), de T. Meyer (Zwiespalt in der marxschen emantipations theorie, Scriptor Verlag Kronberg, 1973), de A. Fränzki (Der misverstandenee Marx, Verlag O. Neske, 1980), de M. Lange (Das Arebeitsprinzip, Verlag ullstein, 1980).

4. Parlant de la situation de base de la pensée de Marx, Marcuse pouvait noter qu'elle correspond à " une nouvelle position révolutionnaire qui gagne une nouvelle vue sur le tout de l'être social et ouvre avec une nouvelle compréhension de la réalité la possibilité d'une action révolutionnarte radicale ». (Beiträge zu einer phänomenologie des bistorischen materialismus, Verlag, VSA, 1974). 
MARX ENTRE LA MÉTAPHYSIQUE ET LA SCIENCE

La tradition marxiste a vu, dans sa grande majorité, en Marx le fondateur d'une nouvelle science. Théoricien de la lutte de classe, du mouvement du devenir historique, de l'avènement du socialisme, Marx est pour elle, celui qui est venu produire la connaissance enfin positive de l'histoire. Le matérialisme historique est le titre de ce nouveau discours scientifique gagné sur la métaphysique et les spéculations passées sur l'histoire'. Si la théorie de Marx s'est en effet historiquement orientée en direction de cette fondation d'un discours de science, la Pensée de Marx quant à elle ne se situe pas et ne s'est jamais située, sur le terrain d'une telle compréhension positive du savoir. Héritière des distinctions opérées par la philosophie idéaliste allemande, distinction kantienne entre le penser et le connaître, hégélienne entre l'entendement et le spéculatif, la pensée de Marx s'inscrit dans la lignée de ces pensées qui ont refusé d'adhérer sans écart à la forme positive du savoir et ont opposé une résistance à la domination du dogmatisme et de la métaphysique positive. Loin qu'elle vise simplement à produire la vérité de ce qui est, elle se veut la pensée d'un projet qui consiste précisément à renverser ce qui est, à en disqualifier la pseudo-valeur de vérité. Pensée aux procédures étrangères à toute visée de connaissance positive dont Marx en 1843 écrivait - " son objet est son ennemi qu'elle veut non pas réfuter mais anéantir »- elle définit une attitude de pensée à l'opposé de toute volonté positive de savoir. Une telle attitude n'a rien d'arbitraire, elle est commandée par ce qui fait proprement l'objet de pensée de Marx, la révolution. La révolution en effet est cet objet particulier qui n'est plus proprement objet positif, positivité à connaître mais projet à penser, réalité qui n'est plus de l'ordre des objets connaissables comme tels, qui n'appartient pas à la classe des objets de l'expérience mais relève d'un domaine de réalités qui s'apparentent à ce que Kant nommait idées transcendantales, objets qui ne peuvent être connus mais seulement pensés. C'est cela que toute la tradition marxiste n'a jamais su reconnaître dans son aveuglement quant à la question de la révolution dont elle ne s'est jamais donné la peine de réfléchir la portée, la prenant toujours

5. L'interprétation althusserienne en a donné le modèle de systématisation. Marx venant ouvrir le continent histoire à la science. 
pour une question déjà résolue, la révolution constituant pour elle un objet non problématique, donnée évidente de la théorie, " factum » ne méritant aucune interrogation mais s'imposant à la théorie comme l'un de ses présupposés dans l'évidence du bien connu $^{6}$. Or c'est la problématicité même de la révolution qui fait l'objet de la pensée de Marx, objet d'une pensée dont le travail ne signifie plus simplement connaître, activité d'enregistrement de la positivité, mais mise question du monde, procès de son questionnement radical. C'est à indiquer des voies possibles d'accès à la profondeur de ce questionnement que nous voudrions contribuer, en définissant trois axes d'interrogations qui traversent la pensée de Marx et nous semblent seuls capables de lui rendre, en 1983, une actualité effective, c'est-à-dire d'en faire à nouveau une puissance efficace de critique de toutes les puissances mondaines qui contribuent aujourd'hui au maintien du statu quo. Il nous faut alors définir la pensée de Marx dans le système d'une triple thèse. Marx, en effet, ne nous semble faire sens aujourd'hui que pour autant qu'il peut être le nom d'un triple mouvement de pensée qui nous oblige à affirmer contre les évidences les mieux établies que loin que sa pensée définisse un quelconque matérialisme, qu'il s'agisse du matérialisme d'une philosophie, de celui d'une théorie de l'histoire ou d'une critique de l'économie politique, elle présente tous les caractères d'un idéalisme radical qui dans sa radicalité même définit à la fois un an-archisme et un individualisme sans compromis.

\section{MARX ET L'IDÉALISME}

Marx est l'héritier de l'idéalisme allemand. L'orthodoxie marxiste n'a cessé de le rappeler contre tous ceux qui essayaient d'effacer en Marx le lien profond qui fait que sa pensée s'est nourrie et définie en partie contre la tradition philosophique

6. Pour la tradition marxiste, la révolution est un événement qui est inscrit dans la loi de la nécessité de l'histoire. Elle définit un apriori de la théorie qui fait du projet révolutionnaire une donnée et non un objet de réflexion. Chez Marx la situation est tout autre. Au départ de sa réflexion, la question de Marx est précisément celle des conditions de possibilité de la révolution. Dans le problème qui occupe tous les jeunes hégéliens et qui a pour nom la réalisation de la philosophie, ce qui est en question n'est rien d'autre que la problématicité de la révolution. $\grave{A}$ quelles conditions la philosophie peut se réaliser, tels sont les termes sous lesquels la pensée de Marx prend forme. Cf. le livre de Thomas Meyer (op. cit., p. 31 ). 
allemande. Mais héritier de l'idéalisme allemand, Marx ne l'est pour elle qu'en ce sens précis qu'il est le philosophe qui a su sortir des apories de la pensée idéaliste pour retrouver le sol positif du matérialisme. 'Marx est celui qui a rejoint la rationnalité du matérialisme sans perdre pour autant, 'comme le fera Feuerbach par exemple, tout ce que l'idéalisme avait pu produire de rationnel. Cette vision des choses où Marx dans un renversement de l'idéalisme et le rejet de la spéculation aurait fondé dans la rationnalité récupérée de l'idéalisme un matérialisme inédit portant enfin la philosophie matérialiste à son concept rationṇel s'est imposée massivement comme préalable à toute discussion raisonnable sur Marx ${ }^{7}$. Une telle vision est pourtant ce qui bloque complètement le sens de la question du rapport de Marx à l'idéalisme allemand, en déportant la recherche vers le champ clos des problèmes qui agitent les réflexions qui s'ordonnent au partage classique de la philosophie en idéalisme et matérialisme, autour duquel ce rapport doit être défini. Le problème de l'idéalisme dans la pensée de Marx n'est pas celui, métaphysique, d'un primat ou non de l'Idée, mais ce que la pensée du procès de l'Idée portait en soi de révolutionnaire, pensée qui chez Hegel venait condenser toutes les contradictions et toutes les apories qui travaillent la problématique de base de l'idéalisme allemand, celle de la subjectivité pratique et de la liberté ${ }^{8}$. Enjeu théorique qui ne se laisse plus définir dans les

7. L'école de Francfort fournit un bon exemple de ce blocage, elle qui a continué à rattacher la pensée de Marx à l'univers de sens du matérialisme. Malgré tous ses efforts pour rétablir à l'intérieur de Marx la substance critique, elle est toujours restée dans les limites que fixait le préjugé matérialiste (Horkheimer, Adorno, Marcuse et dernièrement Habermas ne parviennent pas à rompre totalement le lien au matérialisme qui survit sous la forme d'un primat non liquidé de la nature), aveugle au fait que ce dernier est le verrou qu'il faut faire sauter. Contre les affirmations explicites de Marx, dans La sainte famille ou L'idéologre allemande, où la spéculation philosophique est dénoncée au nom d'un passage à la terre ferme du savoir positif, de la science et du matérialisme, il faut rapporter la pensée de Marx à l'unıvers de sens et au champ de pensée qu'ouvre l'idéalisme allemand, l'idéalisme de l'activité, cet idéalisme auquel Marx dans la première thèse sur Feuerbach se réfere.

8. L'idéalisme allemand est ici vu comme pensée problématique de l'autonomie. La pensée de la révolution est la radicalisation de la problématique directrice de l'idéalisme allemand, la problématique de la liberté. Dans la continuité des réflexions de Kant et de Hegel, Marx définit les finalités de la révolution par la visée de réalisation de la subjectivité libre autonome. Mais à la différence de Kant et de Hegel, il ne soumet plus la valeur de base de la problématique de la liberté à la valeur de sens de l'universel. La radicalisation de l'idéalisme consiste dans cette désimplication de la problématique de l'autonomie de la thématique de l'universel, corrélation qui renvoyait toujours le concept de la liberté à un formalisme abstrait. Chez Kant, le formalisme de la moralité, la liberté ne trouvant à se réaliser concrètement que dans la sphère abstraite de la moralité, sphère séparée de la réalité 
termes simples d'une redéfinition matérialiste de la dialectique hégélienne mais oblige à ne plus limiter le rapport de Marx à l'idéalisme allemand à la seule figure de Hegel mais, dans un élargissement de perspective, d'y impliquer les philosophies de Kant et de Fichte. Remise en perspective de tout l'idéalisme allemand selon un mouvement de multiplication et de complexification des références où ce ne soit plus seule, de façon restrictive, la pensée hégélienne qui se voit prise en compte mais aussi celles de Kant et de Fichte et celà dans un rapport complexe où ces références se croisent et réagissent les unes sur les autres. Dans une telle redéfinition de l'idéalisme allemand, la pensée de Marx ne nous renvoie plus aux questions qui ont occupé la tradition marxiste, obnubilé toute sa pensée, mais apparaît comme la tentative de réarticuler sa problématique directrice en portant à ses dernières conséquences ce que cet idéalisme retenait en soi d'anti-mé-taphysique, en en radicalisant le procès de critique de la métaphysique. Idéalisme ne signifie plus ici primat de l'idée sur la nature, ni même philosophie de la conscience, ni même encore inversion du rapport sujet/prédicat. En dehors de l'univers de sens où ces oppositions se déploient, il renvoie la pensée de Marx comme son procès de radicalisation, comme une forme de sa radicalisation. La pensée de Marx semble alors se définir comme un idéalisme radical. Un tel mouvement de pensée n'a pas échappé à certains interprètes qui sous l'apparente simplicité d'une appartenance de Marx au matérialisme ont bien vu que se tenait la complexité extrême d'une pensée qui, en certains de ces mouvements, va jusqu'à définir un idéalisme plus radical que celui du plus idéaliste des idéalistes, Hegel en personne. G. Haarscher, dans son livre L'ontologie de Marx a montré la profondeur de cette complexité et le nœud des contradictions

à laquelle Hegel venait opposer la sphère historique de la Sittlichkeit autorisant, elle, une médiation effective entre l'universel et le concret. C'est cette solution dont la figure de l'état est la concrétisation historique que Marx soumet à la critique, la liberté hégélienne définissant, tout comme celle de Kant, une forme de liberté abstraite, fausse réalisation de l'autonomie. Le retour à la liberté concrète ne peut se faire que par l'abandon préalable de la valeur de sens de l'universel qui de Kant à Hegel se subordonnait l'idée de la liberté. La radicalisation de l'idéalisme chez Marx consiste alors à libérer l'idée de liberté de l'idée d'une réalisation de l'universel, idée que Marx reconnaît comme mystificatrice et faisant absolument obstacle à toute réalisation possible de la liberté. L'individu libre autonome ne se définit plus comme réalisation concrète de l'universel, mais l'inverse, contre toute forme de subsomption à l'universel, et à la logique qu'elle définit comme autonomie prátique s'arrachant à l'horizon de l'universalité pour revenir à sa détermination singulière, sur le terrain concret de l'individualité de l'individu. 
qui la traverse. Il note que " les critiques adressées à Hegel par le jeune Marx ", loin de se référer au matérialisme ", peuvent se comprendre comme une volonté de radicaliser l'hégélianisme, d'aller plus loin qu'Hegel lui-même sur le chemin tracé par Hegel, d'être plus cohérent que lui au nom d'un indice de rigueur qu'il aurait institué . . . ». Si cela est juste, la radicalisation de Hegel par Marx ne définit pourtant pas un hyperhégélianisme au sens d'une philosophie absolue de la conscience et de l'universel. C'est au contraire contre l'universel que se fera précisément la radicalisation de l'idéalisme allemand, contre l'universel reconnu par la pensée de Marx comme une des valeurs de sens fondatrice de la métaphysique ${ }^{9}$. Donc loin que l'idéalisme radical de Marx équivale à un spiritualisme absolu - spiritualisme qui consiste à vouloir universaliser, en le spiritualisant totalement, l'homme concret et pratique - il se fera procès de critique radicale de toute position de l'universel, critique au nom de la valeur de base de la pensée de la révolution, la subjectivité pratique. De ce point de vue, Régis Debray lorsqu'il note que la pensée de Marx vise la réalisation du sujet comme cause de soi, donc la concrétisation de l'idée centrale de l'idéalisme allemand, à savoir l'autonomie ${ }^{10}$, est au plus près de ce qui est en question même si c'est pour renvoyer un tel projet, où il reconnaît très justement la forme d'une visée anarchiste, au problème insoluble d'une " quadrature du cercle »" Dans Critique de la raison politique il écrit : " Le renversement supposé de la philosophie idéaliste marque en fait l'achèvement du parcours métaphysique l'énigme idéaliste résolue par et dans l'histoire, comme histoire de la réappropriation de l'objet par le sujet, ou de la nécessité extérieure par la liberté subjective. La révolution de Marx a consisté à donner la forme d'un procès historique et une modalité pratique au fantasme théorique originaire : Le sujet comme cause de soi. L'image de l'Homme-Dieu est décroché du cosmos pour passer dans l'histoire, il n'y a rien de plus dans la société com-

9. Cf. note précédente.

10. «Au fond de cette société (le communisme) il y a le vœu tenace et fou que l'homme soit Dieu, acte pur, volonté rationnelle et inengendrée, ne tenant son être que de soi-même et le retenant tout entier dans son creux ". (R. Debray, Critique de la raison politique, Paris, Gallimard, 1982, p. 296).

11. “Impossible de s'associer sans s'assujetir, de s'activer sans se faire passif, de s'invertir sans se dessaisir, telle est la vie sans fin du politique ". (Le Scribe, Paris, Grasset, 1978, p. 77). 
muniste que la réalisation par l'homme de la faculté qu'il a de s'autodéterminer $»$ (p. 295). Réalisation pratique de l'idéal de l'autonomie de l'idéalisme allemand, c'est cela qui fait tout l'objectif de la pensée de Marx, objectif qui n'est pas, comme l'affirme Debray sur la base d'un ensemble de présuppositions anthropologiques, le phantasme d'une raison délirante faisant abstraction " des conditions d'existence d'un ordre politique " (p. 296) mais l'objectif pratique que la révolution a à réaliser pratiquement, exigence minimale à laquelle doit satisfaire tout projet révolutionnaire s'il veut échapper au destin tragique de toutes les révolutions passées, la reconduction du donné à sa puissance seconde dans un mouvement de simple circulation des élites.

L'idéalisme porté à sa radicalité dans la pensée de Marx c'est donc ce mouvement de pensée qui a trouvé ses premiers commencements avec la révolution copernicienne de Kant et qui s'est poursuivi de Kant à Hegel, en passant par Fichte, comme un procès de critique radicale du dogmatisme, procès qui à être réarticulé selon les enjeux qui en déterminent le mouvement profond, se constitue comme procès de destruction de la métaphysique et ouverture d'une pensée de la subjectivité pratique. En effet loin de coïncider, comme Heidegger l'affirmait, avec l'accomplissement moderne de la métaphysique, l'idéalisme allemand et la pensée de la subjectivité qui l'habite constituent le moyen de son dépassement effectif ${ }^{12}$, le principe d'une pensée non métaphysique qui, dans sa structure de base, est une pensée an-archique (qui conduit le procès de déconstruction de toute position de l'arché, la valeur fondatrice de tout l'édifice de la métaphysique). Cet idéalisme porteur d'une critique radicale de la métaphysique, Hegel en formule l'énoncé dans la Science de la logique en ces termes : "La proposition que le fini est idéal constitue l'idéalisme. L'idéalisme de la philosophie consiste seulement dans le fait de ne pas reconnaître le fini comme être vrai. "L'idéalisme radical de Marx, c'est cet idéalisme défini par Hegel

12. Cf. Lebrun, (Kant ou la fin de la métaphysique, Paris, A. Colin, 1970), et La patience du concept, Paris, Gallimard 1972), Theunissen (Sein und Schein, Suhrkamp, 1977), Longuenesse (Hegel et la critique de la métaphysique, Paris, J. Vrin, 1981), W.H. Walsh (Kant's criticism of metaphysics, ed. 
mais libéré de la folie du système, libéré de ce qu'Adorno ${ }^{13}$ pouvait dénoncer comme dictature de la totalité, violence de l'universel, c'est-à-dire libéré de son fantasme de maîtrise absolue, ce fantasme qui est, en la pensée spéculative, comme l'effet d'un dernier sursaut de l'ententement, mouvement de dénégation de la vérité qu'elle met pourtant à jour, l'impossibilité logique/ontologique de la clôture. L'déalisme dans ce contexte équivaut à la destruction de l'arché et de toute la chaîne des concepts qui s'y rattachent.

Critique du fini, l'idéalisme est plus qu'une pensée du mouvement, plus qu'une dialectique telle que le sens commun en fixe le sens, idée qui ne dépasse pas la forme d'une simple métaphysique du mouvement (Cf. Engels). En effet si la pensée idéaliste définit une raison qui procède à la remise en mouvement de tout ce qui s'est figé en positivité, c'est un mouvement de fluidification des solides qui ne se réduit pas, ne se résout pas à mettre simplement les choses en mouvement mais les dissout dans leur prétention à définir des causes absolues, en somme à être le principe de leur propre fondement. Mouvement logique qui défait toute position stable, toutes les positions d'un référent extérieur ou d'une réalité objective hors question, l'idéalisme radical est mouvement pratique de déposition de toute transcendance. C'est en ce sens qu'il faut entendre la critique du fini, elle est puissance de guerre contre la rationalité finie, guerre à la finitude de la raison et à tous ces produits, à commencer par toute position absolue du positif. Pensée qui refuse l'effectivité au fini, le fini c'est-à-dire le positif fixé sur la certitude de sa positivité, l'idéalisme radical montre que le fini séparé de ce qui le pose est une abstraction de la métaphysique à laquelle il faut opposer, comme sa vérité, l'infini du procès par lequel lui, le fini, seul a pu trouver existence. Dans sa critique radicale de la pensée positive qui est savoir fini du fini ${ }^{14}$, incapable de reconnaître la finitude de ses objets, l'idéalisme radical découvre le dispositif présent du monde comme le résultat d'un procès de position par où il a trouvé sa forme actuelle.

13. Cf. T. Adorno (La dialictique négative, Paris, Payot, 1981, Drei studien under Hegel, Suhrkamp, , Zur Metakritik der Exkenntnistheorie, Suhrkamp, ).

14. Theunissen, op. cit., p. 140 et sq., p. 267 et sq. 
L'idéalisme radical est cette pensée qui dispose le monde non plus en fonction de la représentation et de toutes ses fixations de sens mais selon l'ordre de la pratique et qui comme tel ne permet plus que le sujet se laisse fasciner/capter par le miroir de la représentation et fait qu'il redécouvre toujours derrière tout ce qui se donne comme transcendance la marque de la subjectivité qui en est le principe, le travail d'un ensemble de pratiques par où seul ce qui se donne dans l'apparence d'une causa sui a pu être constitué. Contre la métaphysique et ses opérations d'hypostases qui consigne l'ordre du monde comme un ordre intangible et par le mouvement de relativisation des valeurs de sens de la positivité, l'idéalisme radical définit plus qu'un simple mouvement de pensée, il a proprement une signification pratique éminente, celle de constituer un premier moment de déstabilisation du monde, premier moment de sa subversion, subversion que la révolution se donne pour but de conduire à son terme. La critique du fini libère en effet le sujet de son aveuglement sur l'horizon borné de la positivité, elle crève l'écran qui en limitait le mouvement et renvoyant le dispositif présent du monde à sa contingence historique radicale lève cette puissance massive qui jusque là possédait pour lui la force d'une nécessité. Libération de la subjectivité de son impuissance à transcender l'ordre donné du monde qui est aussi libération de la possibilité effective de son affirmation absolue contre toutes les puissances qui jusque là lui faisaient violence. Réveillant dans l'apparente plénitude de la positivité le mouvement de la subjectivité pratique qui l'habite et l'a constituée — « le métabolisme qui opère la réalisation du réel $»-{ }^{15}$ l'idéalisme radical ouvre le monde à sa transformation radicale. Suspendu dans sa validation positive de sens, affecté d'une contingence historique radicale, celle dont l'informe la praxis, le monde abandonne son ancienne position transcendante et découvre sa vraie nature jusque là occultée, le fait qu'effet de pratiques passées, il peut à chaque instant être défait, se voir défaire par une praxis à venir, existence qui derrière l'apparence d'une puissance massive qui à la force d'une nécessité de nature ne définit plus d'une réalité dont le maintien (l'être, la Beständigkeit) est en permanence menacée par la possibilité d'une pratique subversive.

15. Cf. Le très beau livre de Annick Jaulin : La peau du marxisme, Toulouse, Ter, 1979. 
L'idéalisme radical donc en venant faire sauter toute position d'absolu, en dissolvant tout ce qui s'élève au statut d'une transcendance permet de sortir de l'illusion d'un ordre de choses définissant un monde en soi, au delà de toute pratique, au delà de tout mouvement pratique pouvant l'entamer. Il n'y a rien désormais qui puisse prétendre à son indestructibilité, car rien ne peut plus se poser dans la distance infranchissable d'une altérité absolue : Tout est fait et peut donc être défait.

L'ordre des choses et du monde ne se pose plus alors comme la limite indépassable qui définit l'horizon obligé de toute réalité possible. L'idéalisme radical en renvoyant le monde, en le restituant au système des pratiques qui en sont le principe constitutif, recentre le monde sur lui-même et dans ce mouvement de réinscription en soi libère tous les possibles qui dans le geste de son institution et conformément à la règle de toute instauration d'un ordre se sont vus soumis au procès d'une négation radicale. En effet, comme le note $R$. Schürmann, " chacune des diverses positions fondamentales de l'humanité occidentale au milieu des étants amène sa propre économie qui d'abord s'impose eux étants pour ensuite, durant une période qui est la sienne, en disposer. L'imposition et la disposition express l'arché de ces positions fondamentales . . . Le geste fondateur d'une cité inaugure le champ des possibles dont elle vivra et le commande. En tant qu'il le commande, un tel geste décrète le code qui restera en vigueur à travers ce champ » (p. 48) Ce décret est aussi loi d'exclusion, il interdit. Dans le mouvement même d'ouverture du champ des possibles dont il vivra, l'instauration d'un ordre, en effet, opère dans le même temps la clôture de l'horizon infini du possible en excluant tous les autres possibles qui pourraient en menacer l'économie principielle. Cette exclusion s'opère en faisant de ces possibles des impensables, en les renvoyant au domaine d'une pure impossibilité. La révolution n'est plus alors contrainte de trouver son principe dans le cercle fermé de la causalité linéaire de la positivité, se condamnant toujours à ne pouvoir que reproduire celle-ci. Idéalisme radical et pensée de la révolution communiquent ici au point le plus chaud de leur fusion où l'idéalisme rejoint dans cet espace libéré, où il n'y a plus de transcendance, plus d'instance possible pour fonder quelque chose comme un en soi, sa vérité concrète, sa déter- 
mination, son identité de pensée an-archique, cette pensée qui est au fondement de la pensée de la révolution de Marx comme de qui la rend possible dans sa radicalité même. La mise en question par l'idéalisme radical du monde hypostasié, la disqualification du fini, c'est en effet celle du geste qui en a autorisé les différentes positions, la disqualification de tout ce qui rend possible toute position absolue d'un fondement, à commencer par la valeur de sens sur quoi s'appuient toutes ces procédures. La critique du fini et de la positivité est avant tout et fondamentalement la critique du référent, de tous les référents, du fondement de tous les gestes fondateurs, critique du principe fondateur de toute métaphysique, l'arché.

Entre le projet de la révolution et l'an-archisme le lien est donc avant tout d'ordre éminemment théorique, la disqualification an-archique de l'arché est la présupposition du libre mouvement de la pensée de la révolution. Dans le dessaisissement du monde de sa transcendance, celle que lui préservait toujours le maintien d'une positivité hors question (celle par exemple dans le marxisme des soi-disantes lois naturelles de l'histoire et du système des nécessités qu'elles semblent pouvoir définir), s'ouvre la brèche par où le projet de la révolution radicale, jusque là bloqué sur l'horizon fermé de la positivité, peut s'attaquer au monde en sa racine. La révolution peut alors ${ }^{16}$ s'inscrire dans toute sa radicalité sur le sol concret de l'histoire, dans la chair même de son mouvement.

La pensée de Marx nous semble bien se tenir dans la proximité de cette pensée an-archique dont $\mathrm{R}$. Schürmann dans son livre sur Heidegger pouvait écrire : «Anarchie dit le destin qui fait dépérir les principes auxquels les occidentaux ont rapporté, depuis Platon, leurs faits et gestes pour les y ancrer, les soustraire aux doutes et aux changements, principe qui fait éclater le nomos de notre oikos, donne congé à la police des marques métaphy-

16. Le concept de la transformation révolutionnaire du marxisme reste enfermé dans le cercle limité d'un simple réagencement des éléments déjà présents. La modification de la réalité se fait sur un fond en soi qui reste lui-même inchangé. Il y a au delà de la praxis et de son mouvement un ordre de l'en-soi qui définit pour eux leur apriori et leur loi. La praxis ne devient effective qu'à se plier à la loi de cet en-soi qui en commande le mouvement et les finalités. Avec l'idéalisme radical, c'est cette altérité qui tombe. Le monde dans sa totalité est suspendu à la pratique à venir sans qu'aucun segment de sa réalité ne lui reste inaccessible. 
siques, à la police des principes épochaux qui sont ce qui donne cohésion, fondement et assurance au monde ». (op. cit. p. 18) Destin qui s'apparente à celui que doit effectuer le procès de la révolution et qui engage le double ordre croisé de la pensée et de la politique. 1) De la pensée, en tant que la pensée de Marx se déploie contre l'idéologie du fondement, c'est-à-dire la métaphysique et tous ses gestes théoriques de fondation, à commencer celui le plus inaperçu parce qu'au principe de notre rapport le plus immédiat au monde (mais cette immédiateté est le résultat de tout un procès complexe de constitution) ${ }^{*}$ et à sa valeur de sens, celui de la positivisation de la positivité, cette position particulière de la positivité qui n'est pas un acte pur de la théorie, ni même une donnée anthropologique de la conscience (la conscience naturelle est toujours produit d'une décision pratique) mais toujours un geste engagé, une prise de position en faveur d'une certaine organisation de la réalité. Or c'est précisément contre cette organisation que s'organise le projet révolutionnaire de Marx. 2) De la politique, contre la materio-praxeo-logie du pouvoir qui est mise en pratique de cette même métaphysique. Il y a en effet implication immédiate et réciproque du philosophique et du politique où comme le note J.L. Nancy "le politique n'est pas plus extérieur ou antérieur au philosophique que le philosophique en général est indépendant du politique ». (Rejouer le politique, Paris, Galilée, 1980) La positivité, sa constitution/institution comme valeur de base de tout discours relève d'une mise en perspective (du monde) qui ne peut prétendre à l'innocence d'un pur reflet, elle est l'effet d'une décision ontologique qui est aussi politique. L'interdiction de l'arché, la disqualification de toute volonté fondatrice dans le renvoi de tout arrangement et de tout ordre à un établissement, au procès d'une constitution, ont une valeur politique éminente car c'est le pouvoir lui-même qui se trouve frappé en son principe. Le pouvoir en effet ne s'instaurait comme puissance effective qu'à travers un ensemble d'opérations d'immunisation par où il élevait sa puissance à la transcendance. Procès de sa propre archeologisation par où il faisait de son ordre naturel et de la constellation finie des choses et des hommes qu'incarne le monde

* Cf. F. Borkenau : "Der Uberganf von feudalen zum burgerlichen Weltbilds " 16/17 Darmstadt 1971. 
une véritable nature. Or c'est cette double procédure, naturalisation du monde et, dans celui-ci, de l'ordre policier qui y règne, procédure vitale de légitimation, qui avec la pensée anarchique de Marx ne se laisse plus opérer. La pratique de déstabilisation du monde, détruisant les images positives du monde, prive de leur base tous les discours de légitimation de l'ordre des choses et en tout premier le discours fondateur de tous ces discours, le discours que la réalité, la positivité tient sur ellemême, le discours même du monde, ce discours muet que le monde nous tient et qui le renvoie à une innocence postulée des choses. Opération critique essentielle dans ce travail contre l'effet de naturalité des choses, contre les effets de sens qui font que le monde à la force des choses ${ }^{17}$ puisque c'est l'illusion au principe de toutes les illusions, l'idée même de cette force des choses et de leur innocence qui se voit détruite, idée qui n'est jamais rien d'autre que le résultat de l'exclusion de la praxis dans la structure d'arrangement des choses et des hommes, de l'occultation, de l'évacuation de la volonté qui a décidé de cette architecture de base de la réalité. La structure d'arrangement du monde, ainsi rapportée à un principe d'organisation toujours en dernière instance subjectif, ne peut plus s'arracher à sa nature d'ordre voulu et se poser comme indépassable, ordre du monde qui vaut de toute éternité, fond naturel qui ne relève d'aucun établissement et défini proprement une fatalité. Ouverture d'une béance, d'un espace sans fond où c'est non seulement le monde et l'ordre qu'il définit qui sont renvoyés à leur précarité de base mais aussi le principe de sa police, le pouvoir, qui n'a plus rien pour se fonder, plus rien pour se légitimer, plus rien que la contingence et l'arbitraire radicaux de son auto-établissement. ${ }^{18}$

17. Il y a là un des effets majeurs de ce que Marx reconnaît sous le concept du fétichisme : c'est sous la forme de choses, d'un monde de choses que se donne immédiatement l'ensemble du procès de la réalité sociale. Dans sa forme phénoménale il correspond à un renversement d'un monde de simple choses identique au monde naturel. Ce premier effet objectif se voit redoublé et confirmé dès lors que le monde est pris en vue par une pensée qui se règle sur la loi de la représentation. C'est à cette loi de la représentation et à tous ses effets de sens que s'oppose de façon radicale l'idéalisme qui vient briser l'illusion mystifiante du pseudo poids naturel des choses et sa conséquence pratique immédiate, la paralysie des sujets historiques qui s'y confrontent. L'idéalisme en brisant la familiarité de notre rapport au monde permet de rejoindre une transparence qui rend à nouveau sensible la violence masquée qui est au fondement de ce qui nous semble le plus propre et le plus familier.

18. A. Gorz dans Adıeux au prolétariat note : "Marx disait que les capitalistes en (il s'agit de la loi du capital) sont les fonctionnaires : à la fois oppresseurs et aliénés, ils subissent 
Renvoi du pouvoir et de son ordre à eux-mêmes, destruction définitive de toute instance et de toute transcendance auxquelles ils puissent trouver référence, qui invalident le pouvoir dans sa prétention à la légitimité et le laissent alors apparaître dans la nudité sans voile de son être vrai comme le pur rapport de force qu'il est, violence faite aux individus que rien ne saurait plus justifier. C'est contre cette violence faite aux sujets concrets de l'histoire que Marx pense le projet de la révolution, qu'il réclame la subversion radicale de toute la réalité, la destruction de l'état de chose existant. Le pouvoir renvoyé au vide de son fondement, la disqualification de l'arché ouvre à la pensée d'un individualisme qui définira les conditions d'impossibilité de l'oppression en développant les conséquences pratiques de la position anarchique du penser qui est libération de la liberté. C'est là tout le projet de pensée de Marx, projet d'une pensée qui lie la disqualification de la valeur de sens de l'arché à une problématique de la liberté dont le concept renvoie à une pensée révolutionnaire de la subjectivité qui ne se laisse plus réduire à la figure bourgeoise de l'individu mais, contre celle-ci, vise à libérer la figure historique de l'individu de ses contradictions.

\section{L'INDIVIDUALISME}

On a récemment affirmé que la pensée de Marx contrairement à ce qu'il paraissait, appartenait à l'aire des pensées libérales. Contre toute apparence Marx serait l'héritier de la pensée libérale, l'héritier d'une problématique refusant la politique

une loi scéllée dans les choses et la transmettenit. Ils administrent le fonctionnement du capital, il ne le commandent pas. Ils ne possèdent pas le pouvoir, ils sont possédés par lui. Le pouvoir n'est pas sujet : il est système de rapports, c'est-à-dire structure . . C'est cette dilution à l'infini du pouvoir dans l'ordre des choses qui donnent à ses détenteurs leur légitimité ... Les porteurs du pouvoir ne commandent aux hommes qu'au nom d'une soumission à un ordre des choses donné et dont nul ne se reconnaît l'auteur . . . Le pouvoir est un effet de système, il résulte de la structuration d'un système matériel de rapports dans lequel un loi des choses asservit les hommes par l'intermédiaire d'autres hommes ". (Paris, Seuil, 1980, p. ) Cette vision des choses n'est juste qu'à ne pas oublier que la loi scellée dans les choses, la loi des choses qui asservit est elle-même un effet, effet de l'institution d'un ordre des choses par le truchement d'un procès de l'ordre des pratiques, que la naturalité de la loi n'est que le résultat de son caractère pratique, de l'oubli qu'elle est institution subjective. En renvoyant l'ordre des choses et le système des lois qu'il semble définir comme une loi de nature au procès de son institution dans l'ordre des pratiques, l'an-archisme prive le pouvoir de toute naturalité, le renvoie à sa particularité, cela même qu'il travaille continament à effacer pour assurer son autorité. Le pouvoir ainsi dépouillé de sa naturalité de son apparent enracinement dans une loi naturelle des choses découvre son sans-fond fondamental. 
comme instance séparée et trouvant dans le cœur même de la société le principe de son unité, de son unification. Dans cette appartenance où Marx serait venu reprendre à son compte l'utopie économique libérale se tiendrait les germes du totalitarisme que l'histoire du marxisme allait laisser éclore en quelques uns des points de son application. L'illusion d'une société transparente à elle-même, d'une société indivisée et ayant retrouvé son organicité aurait été à l'origine de toutes les aberrations qu'a connues le marxisme. Rosanvallon dans son livre Le Capitalisme Utopique peut noter : "en définissant le communisme comme société immédiate et transparente, Marx finit par concevoir une société complètement abstraite, dans laquelle chaque individu est un résumé de l'universalité, la société n'étant structurée que par un pur commerce entre les hommes . . . Le communisme comme société de marché pure achève donc l'utopie libérale au prix de la constitution contradictoire d'un organisme social total. Les aliénations ponctuelles sont remplacées par une seule et unique aliénation globale : l'homme est contraint à une universalité qui ne peut être réalisée que par une force extérieure . . . Le totalitarisme constitue ainsi le dernier mot de l'utopie de la transparence sociale ». (p. 207)

Ces analyses qui ont mis en évidence les héritages contradictoires qui se partagent la pensée historique de Marx et ont su soulever un certain nombre de questions décisives quant aux problèmes de la révolution et ses finalités ne touchent pas cependant ce que nous avons défini comme l'impensé de Marx, ce mouvement de pensée qui développe la pensée an-archique dont il faut maintenant définir la signification politique concrète. En effet si la philosophie de Marx peut se laisser définir comme une pensée de l'individu, si elle présente bien en soi le principe de l'individualité, cette pensée de l'individu est comme l'a indiqué M. Henry pensée ontologique, une pensée que rien ne permet de rapporter à la vision libérale du monde ni à l'idéologie économique qui restent de part en part descriptives. Renvoyer Marx à une telle vision c'est oublier simplement que Marx été le critique radical de l'univers conceptuel et représentatif de la pensée libérale, qu'il a montré qu'il correspondait à une perception, conception du monde qui dérive d'un point de vue limité et mystifiant. Marx, en effet, depuis sa rencontre avec l'économie politique n'a cessé de dénoncer le point de vue central de la 
vision libérale, le point de vue de la circulation, point de vue structurellement incapable de rendre compte de la structure effective de la réalité qu'il prétend exhiber et qu'il recouvre par le modèle abstrait d'un espace homogène où les individus sont réduits à ne plus définir que les points de croisement sans épaisseur d'un système mécanique de forces. Tout le travail de Marx dans sa critique de l'économie politique a été de récuser ce niveau d'analyse de la réalité pour revenir du plan de la circulation à celui de la production où se tient le noeud de sa pensée individualiste, la subjectivité pratique et les formes de sa structuration aliénée/aliénante sous la loi de mouvement du capital. Le point de vue de l'individualisme de Marx n'est pas et ne reste pas empirique comme celui du libéralisme qui ne parvient à ne définir que la figure abstraite de l'individu égoöste telle qu'elle se construit dans la fiction de l'utopie de marché, figure sans contenu réel qui est le reflet théorique de l'image que s'en font les agents de l'histoire au niveau de leur représentation idéologique. Concept ontologique, concept social d'une réalité ontologique de base qui est la subjectivité pratique, l'individu renvoie au delà de l'empirie de l'économie, au delà de la réalité que définit le procès de sérialisation par quoi le capital sur le terrain de la réalité sociale et des rapports sociaux renvoie chaque sujet à une individualité abstraite et vide, à une dimension de réalité qui correspond à la structure de base de la subjectivité telle que Marx en hérite la pensée problématique de l'idéalisme allemand et telle qu'il cherche à en penser les conditions de réalisation effective dans la matérialité du devenir historique. L'individu et son concept, loin de trouver leurs références dans le monde de l'économie, les prennent dans une réflexion sur la structure de la subjectivité, réflexion orientée par la question de l'autonomie pratique, d'une part, et par celle de la révolution, de l'autre. L'individu de Marx ne se définit donc pas plus par l'anthropologie que par l'économie, il est de part en part produit de l'histoire et produit contradictoire qui nous renvoie à une double référence, une référence théorique, la problématique de la révolution et de la liberté telle que l'idéalisme allemand l'a mise en place, et une référence pratique, la réalité du destin ${ }^{19}$

19. De ce point de vue l'individu tel que le définit la pensée libérale reste à jamais incapable à définir une puissance en mesure de résister et de faire obstacle à la violence qui accompagne ce destin. 
de l'individu sous la loi du capital. La pensée du communisme n'est rien d'autre que la pensée de cet individu.

Le passage du communisme n'est pas alors ce passage, qu'imagine le marxisme, de la société divisée par ses contradictions à une société unifié qui a supprimé à l'intérieur de soi cette contradiction de soi à soi, passage inscrit dans la loi même d'évolution de la contradiction de base du mode de production capitaliste. En effet si la révolution s'articule bien sur une contradiction du capital, ce n'est plus sur celle entre le développement des forces productives et l'état des rapports de production, mais sur celle que recouvre précisément le destin que connaît la subjectivité sous le régime du capital et qui nous renvoie au lieu refoulé de la subjectivité. Aussi si la société bourgeoise se laisse bien définir, avec J.L. Petit, comme " entendement, division, quantification, indifférence » on ne peut l'opposer comme cet auteur le fait à une définition du communisme comme « communauté, ... synthèse, totalité, organicité retrouvée, coalescence des membres du tout ». (op. cit. p. 244). Le communisme n'est pas comme tel pensée de la communauté, mais pensée de l'individualité (pensée radicale de l'individualité et pensée de l'individualité radicale) et la question du communisme est prioritairement (il s'agit d'une priorité problématique et ontologique) question non pas du mode de socialisation de la société ${ }^{20}$ mais problème du principe d'individuation du sujet à l'intérieur de la société capitaliste, principe qui du point de vue critique ne produit et ne peut produire qu'une forme abstraite et vide du sujet : le sujet atomisé, borné, limité. L'individu produit par le système du capital est en effet un individu réduit. à sa pure forme abstraite, individu nié dans son individualité, référé et prisonnier d'un système d'individuation qui ne lui attribue qu'une identité sans contenu ; tous les sujets sont posés comme les unités équivalentes d'une seule et unique masse, réduction de l'individu à un nombre. L'économie est le terrain de cette égalisation atomistique des sujets historiques qui en fait des

20. Rosanvallon renvoyant Marx à Locke peut écrire : « le but du communisme c'est de rendre la maîtrise de la société civile, confisquée par la bourgeoisie qui en a fait sa propriété privée dans le capitalisme, à la société toute entière ». (op. cit. p. 35). 
sujets fonctionnalisées à des fins étrangères et aliénantes, fonctionnaires d'une logique qui ne signifie rien moins que leur propre suppression comme individus vivants, simples accidents, accessoires et purs instruments de la loi du capital.

C'est à partir de cet état de fait qui est comme l'écrivait Marcuse « catastrophe de l'essence humaine " que se pense le communisme comme résultat d'un procès de radicalisation subversive du procès d'individuation contradictoire des individus, radicalisation révolutionnaire de l'autonomisation du sujet qui ne se fait plus alors selon les exigences aliénantes de la logique atomisante du capital mais en vue de la réalisation de la puissance jusque là retenue de l'autonomie, en vue de la réalisation enfin effective de la liberté cette fois libérée de son procès d'instrumentalisation par le capital et se posant comme sa propre finalité, véritable Selbstzweck : réalisation concrète de la liberté qui est en même temps réalisation effective de l'individualité.

L'individualisme de Marx part donc de l'individu tel que le mode de production capitaliste le produit. Si l'individu, tout comme la liberté, sont des formes que le capital porte à l'existence historique, cette production s'est faite et s'opère dans la plus profonde des contradictions. L'individu tel que le définit le système du capital - la définition capitaliste de l'individu le finitise sur les fins externes du capital - tout comme la liberté qui y correspond ne sont que des caricatures de la puissance dont ils sont l'expression aliénée. Le capitalisme est à la fois la forme historique où émerge la réalité de l'individu comme forme de l'être-là du sujet historique et mode de production de la vie où cette émergence s'opère sous un mode d'être de l'individualité qui en est la négation radicale. Il définit comme tel un mode de production contradictoire de l'individualité où sont produites à la fois les conditions sociales de réalisation de l'autonomie et l'impossibilité de la réaliser. L'individu vivant sous le capital est pris dans la contradiction entre la réalisation de sa vie, l'activité libre, et la nécessité de ne pas la réaliser, le travail et la vente de force de travail, pour la conserver. Ce que Marx pouvait écrire du travail dans les Grundrisse - " son procès de réalisation est procès de déréalisation du travail. Le travail est objectif, mais il crée l'objectivité comme son non-être à lui ou comme l'existence de sa non-existence " (Fondements de la cri- 
tique de l'économie politique, Paris, Anthropos, 1968, t. 1, p. 417) - s'applique avant tout à l'individu et son destin. Sous le règne du capital, l'individu n'est plus que sujet assujetti, sujet soumis, aliéné à un système de production de l'aliénation. Mode de production de l'individualité bornée, le capitalisme est, de la même façon, moment historique de la production de la liberté sous la forme de la non liberté, liberté formelle qui ne connaît qu'une existence aliénée, liberté abstraite de soi et pliée à la reproduction infinie de l'ordre du capital, c'est-à-dire à la logique de sa soumission à une fin étrangère. C'est ainsi que Marx, toujours dans les Grundrisse pourra parler de la liberté sous le régime de domination du capital comme d'un " développement libre sur une base bornée » qui est « en même temps la suppression la plus totale de toute liberté individuelle où l'individualité est complètement subjugé par les conditions sociales qui prennent les formes de puissances matérielles et même d'objets supérieurs, la forme de choses indépendantes des individus mêmes et de leurs rapports (id. t. 2, p. 188).

Individu qui a revêtu la forme de sa négation, liberté qui se pose sous le mode de la non-liberté, ce sont ces contradictions là, contradictions qui retiennent tout le concret du projet de subversion du monde, qui font le problème de Marx. Le communisme n'est rien d'autre que cette visée pratique de subversion de l'ordre du monde en vue de réaliser ces puissances niées dans le système du capital : libérer la liberté de son instrumentisation par le capital, porter l'individu abstrait du capital à sa détermination d'essence, à sa détermination de sujet libre autonome, de puissance pratique autonome. Le communisme ne se laisse plus définir comme une pensée de la communauté et nous sommes loin ici des problèmes d'unité réalisée du social, de transparence sociale. Si il y a dans la finalité révolutionnaire une visée de transparence, ce n'est pas celle de la totalité sociale à elle-même mais celle du sujet à soi, transparence du sujet à son monde où le sujet devient pleinement sujet en reconnaissant en soi la puissance inaliénable de son fond, sa réalité de puissance pratique libre et autonome. Transparence qui signifie donc le retour de l'individu englué à lui-même, auprès de soi ${ }^{21}$, retour de l'in-

21. Cette problématique n’appartient plus à la métaphysique du propre, à la logique identitaire. Il ne s'agit plus dans ce retour du sujet auprès de soi de poser l'idée d'un propre 
dividu dans la positivité du monde à sa vérité de sujet libre autonome, de lieu de réalisation possible de la liberté, de réalisation enfin effective de la puissance pratique de l'autonomie. En effet, la forme de l'individuation capitaliste ne produit jamais qu'une caricature d'autonomie ; l'autonomie capitaliste de l'individu atomisé qui est autonomie privée de sa substance, autonomie habitée, dominée, subjugée par l'hétéronomie absolue d'un système de finalité qui ne travaille qu'à la reproduction de son aliénation et de sa suppression. L'individu du capital, c'est l'individu-atome, or l'atome c'est ce qui n'a pas en soi le principe de son mouvement, ce qui ne se meut que par l'action de forces extérieures, c'est la figure par excellence de l'absence d'autonomie. Rendre à l'instance de l'autonomie du sujet son efficace, son effectivité contre sa fixation dans le système des déterminations hétéronomes de la reproduction sociale, telle se définit la fin de la révolution, une fin qui pour pouvoir se poser présuppose une autre pensée de la finalité que celle qui jusqu'à présent à dominer tous les modèles de la révolution, une pensée de la finalité qui ne se soumet plus la fin au système de ce que R. Schurmann appelle la «téléocratie " et renvoie toujours le projet révolutionnaire au procès d'une construction positive d'un ordre, d'une fondation ou d'un gouvernement social, ces objectifs qui renvoient toujours à un projet de maîtrise contre lequel la pensée communiste se déploie.

qui définirait la norme à quoi doivent se conformer les sujets pour être libres. Le sujet ne doit plus conquérir sa-liberté à l'intérieur du schème theo-logique que à finit la métaphysique humaniste. Il s'agit plus de se conformer à ce qui en l'homme définit son essence, l'humanité. Si le mouvement révolutionnaire vise bien à détruire la finitude du sujet (et non de l'homme, l'homme est une catégorie de la métaphysique humaniste et chez Marx la pensée de la subjectivité ne s'identifie plus à une pensée de l'homme mais elle est pensée de l'individu, ce qui ne permet plus aucun développement de l'humanisme, la pensée de l'individu étant la critique en acte de toute idée d'humanité, le procès de mise à mort de la catégorie de base à laquelle cette idée renvoie, l'universel), il ne s'agit plus de cette finitude métaphysique hétitée de la théologie qui en fait un individu fini par rapport à l'infinité du genre. La finitude à supprimer est celle que lui impose sa normalisation sociale historique sur la loi de son aliénation, cette restriction, ce rétrécissement des possibilités de sa vie, cette violence faite au libre mouvement de ses passions. La problématique du propre s'oppose totalement à cette visée qui est celle d'un laisserêtre de la différence, d'un refus radical de toute violence identificatrice, problématique qui est toujours d'essence moralisante et retient en soi la violence terroriste d'un devoir être qui est impératif absolu, loi. Le retour à soi de l'individu dans le projet an-archiste ne pose plus de norme, elle s'opère dans le respect de l'indépassable de l'individualité de chaque sujet. 


\section{L'AN-ARCHISME}

L'individu du capital, c'est l'individu condamné à un destin qui équivaut à sa dissolution. La révolution communiste n'a pas d'autre objectif qu'à mettre fin à cette violence que connaît l'individu sous le régime du capital et à libérer l'espace du libre déploiement des individus et de ce que Marx appelle leurs forces essentielles. Pour la pensée communiste, il s'agit moins de découvrir une quelconque loi positive du monde nous donnant connaissance du processus objectif de l'histoire dont il s'agirait alors de reconnaître la courbe et de maîtriser les moments décisifs, que de reconnaître le procès de déploiement des aliénations dans l'espace social, de repérer les lieux de la violence et la logique qui la structure pour en défaire l'efficace. Dans cette perspective, le communisme ne peut plus s'égaler à un projet de fondation sociale; il ne peut s'agir pour lui d'établir contre l'ordre ancien un nouvel ordre réalisant la raison à laquelle le premier faisait violence. La violence à détruire est plus radicale que cela, elle touche à l'existence de l'ordre comme tel, à tout projet de fondation d'un ordre. Un tel projet en effet relève pour la raison an-archique d'une pensée habitée d'une volonté pénétrée par la métaphysique, volonté de maîtrise et de pouvoir qui est précisément ce que la révolution an-archique vise à rendre impossible, ineffectif. Fonder, construire, édifier, ce sont des schèmes qui présupposent toujours la pensée archique a leur fondement, le schéma d'une base à établir et à partir de laquelle tout le mouvement de la révolution a à s'organiser et à s'ordonner. C'est un tel réglage que le procès de la révolution et la pensée an-archique refuse en y reconnaissant le signe d'un redéploiement des puissances qu'ils visent à abattre, celles du pouvoir et d'une volonté de maîtrise et de domination sur les sujets. À l'opposé de tout projet de gestion rationnelle du réel, projet toujours d'essence techno-cratique, il est fondamentalement visée d'essence pratique. Il ne s'agit plus de construction positive d'un ordre, mais de déconstruction d'un monde ${ }^{22}$ et dans celui-ci d'un certain mode de structuration de la subjectivité dans la pro-

22. "Le communisme pour nous n'est pas un état qu'il faut créer ni un idéal vers lequel la réalité doit s'orienter, nous nommons communisme le mouvement réel qui aboutit l'ordre établi " (K. Marx, Idéologie Allemande, ). Ce mouvement réel c'est celui de la nouvelle subjectivité que la révolution doit "produire" comme sa condition et sa fin propres. 
duction d'un nouveau sujet. Un tel procès ne se laisse plus penser sous les concepts positifs de la poésie et de la techné, mais est praxis libératrice, praxis au sens non productif mais pratique de ce concept, pratique an-archique de "production " d'un nouveau mode d'être de la subjectivité, libération pratique de la puissance pratique de l'autonomie et ouvertüre d'un espace de déploiement à la réalisation effective de la subjectivité, double mouvement où les individus retournant à leur autonomie constitutive ne se font plus alors agents positifs de la reproduction sociale mais s'affirment comme puissance autonome, réalité effective d'une volonté libre qui ne laisse plus prévaloir que ce qui ne fait pas violence à son autonomie. "Rendre impossible tout ce qui existe indépendemment des individus " pouvait écrire Marx entre 1842 et 1844 , telle est l'exigence à laquelle la révolution doit satisfaire pour être à la hauteur de son principe, le principe an-archique, ce principe pour qui il s'agit de défaire un ordre (non pas d'en construire un), de déstructurer un espace de places et de fonctions et ce qui autorise une telle disposition, l'arché, le principe d'organisation du tout et en tout premier lieu ce qui lui donne son efficace, le rapport des sujets à leur propre existence ${ }^{23}:$ rendre impossible sa reproduction en visant la «production" d'une individualité qui rend innefficace la puissance de l'arché telle qu'elle a toujours fonctionné comme force de soumission et d'oppression de la subjectivité.

La révolution communiste ne définit donc plus une finalité positive mais l'émergence d'une négativité qui doit définir les conditions d'impossibilité d'exercice d'une certaine violence, celle jusqu'ici faite à l'autonomie du sujet. La révolution vise et ne vise que cette autonomie et ses conditions, l'individu libre autonome en constitue l'unique et seule finalité. La destruction du capitalisme dès lors change radicalement de sens par rapport à sa définition traditionnelle. Il ne s'agit plus de produire une nouvelle société, de construire le socialisme, d'édifier-la base de la nouvelle société, ces visées positives qui courent toujours le risque de tomber sous le schème de la pensée technique des fins et des moyens et qui comme telles renvoient la pensée et le

23. "Remplacer la suprématie des conditions extérieures et du hasard sur les individus par la suprématie des individus sur le hasard et les conditions objectives coïncide avec la tâche de donner à la société une organisation communiste " (p. 59). 
procès de la révolution à un problème plus technique que pratique (Lénine en est la figure exemplaire). La suppression de l'ordre du capital ne se fait pas au profit de la production de quelque chose comme une objectivité sociale, son objet/objectif n'est plus de l'ordre d'une positivité à produire, c'est un sujet, la « production " d'une nouvelle forme de la subjectivité, " production " de la subjectivité libre des sujets "production » des producteurs à leur autonomie pratique, à leur puissance d'individus libres autonomes. Cette " production ", la nécessité de ce nouveau sujet, c'est ce que définit en chacun de ses points le projet de la révolution qui s'appuie sur la conception an-archique de l'individu et sur le statut qui lui est reconnu. A. Negri remarque justement que " le communisme n'est ni la téléologie du système du Capital, ni sa catastrophe. C'est un nouveau sujet qui prend forme, qui transforme la réalité et détruit la société. Il est la positivité d'une construction libre de la subjectivité » (Marx au delà de Marx, p. 287). Nouvelle constitution libre autonome du sujet qui est "libération sociale du sujet " où "l'efficacité du sujet libéré-écrit encore Negri s'oppose et renverse l'efficacité mystifiée de la socialisation capitaliste ». (id., p. 273) Le communisme s'oppose en ce point de façon radicale à tous les projets socialistes de fondation d'un ordre, de construction de la nouvelle société qui toujours relèvent de la logique du pouvoir et de la violence archique. Dans la problématique socialiste, il s'agit toujours en effet de poser le fondement et sur ce fondement d'édifier le nouvel ordre socialiste. C'est là l'impératif qui définit la loi de la révolution à quoi tout le mouvement de la transformation doit se plier. Il y a, au centre de cette vision, toujours le même geste archique d'une position fondamentale du fondement, qu'il s'agisse du genre, de l'histoire, du collectif ou du parti, position d'un premier principe transcendant, d'un centre organisateur et d'un principe normatif par rapport auxquels le mouvement des sujets historiques trouve son sens et doit s'articuler. Une telle vision redéploie l'économie du pouvoir ; il s'agit toujours de s'assurer une maîtrise sur le devenir social et les sujets historiques se voient de nouveau fonctionnalisés non plus cette fois aux nécessité du capital et de sa reproduction mais à celles supposées du mouvement de l'histoire : visée qui rabaisse toujours la pratique à la techné, opère 
une véritable archeo-logisation des pratiques, leur normalisation sur une arché dont il faut réaliser l'ordre. La révolution ordonne sa téléologie sur la visée d'une positivité à produire, d'un ordre à constituer, projet toujours habité par une volonté positive de maîtrise qui a pour conséquence la relance de tous les effets aliénants/oppressants de la transcendance: les individus se retrouvent à nouveau transcendés par une fin qui les dépasse et qui se les approprie comme simple moyen. Les sujets de l'histoire sont alors contraints à se définir par rapport à une norme qui devient la référence où chacun doit trouver son identité. Le principe anarchique fonctionne contre cette logique de la normalisation et de l'indifférenciation qui est le principe de la toute première violence faite à ce que Marx reconnaît comme la première richesse sociale, l'individualité. Dans les Grundrisse Marx écrit : " mais que sera la richesse une fois dépouillée de sa forme bourgeoise encore limitée ? Ce sera l'universalité des besoins, des capacités, des jouissances, des forces productives, etc, des individus . . . Ce sera la domination pleinement développée de l'homme sur les forces naturelles, sur la nature proprement dite aussi bien que sur sa nature à lui. Ce sera l'épanouissement entier de ses capacités créatrice, sans autre présupposition que le cours historique antérieur qui fait de cette totalité du développement un but en soi ; en d'autres termes le développement de toutes les forces humaines en tant que telles, sans qu'elles soient mesurées d'après un étalon préétabli. L'homme ne se reproduira plus comme unilatéralité, mais comme totalité. Il ne cherchera pas à demeurer quelque chose qui a déjà été, mais s'insérera dans le mouvement absolu du devenir » (t. 1, p. 450). Vision du communisme dont la finalité directrice est « l'épanouissement entier de l'intériorité humaine " et qui s'oppose à toute volonté d'homogénéisation, d'unification, d'identification normatif, ces gestes qui ne servent jamais qu'un projet de maîtrise. Avec le principe an-archique, plus de volonté de rapporter toutes les différences à l'unité, mais une logique s'apparentant à cette économie aux lois singulières que Derrida délimitait sous le concept de la « différence », principe qui est « non-principe et non fondement ", " qui se dérobe définitivement à l'attente d'une archie rassurante », « ne commande rien, ne règne sur rien et n'exerce nulle part aucune autorité » et est ce qui « n'instaurien et n'exerce 
nulle part aucune autorité » et est ce qui " n'instaure plus aucun royaume mais au contraire fomente la subversion de tout royaume ». (p. 394)

Ce que le projet communiste oppose alors à l'anarchie des intérêts privés dans le système du capital n'est pas l'ordre réglé d'une société libérée de ses contradictions et déployant dans une harmonie retrouvée une parfaite maîtrise de soi $^{24}$ mais ce qui rend désormais intenable une telle maîtrise, l'an-archie des libertés, c'est-à-dire l'ouverture d'un espace établissant la prévalence de l'intérêt de la liberté contre tout établissement possible d'un ordre. Espace du libre déploiement de la subjectivité où les sujets pratiques cessent de se produire comme objet pour enfin s'assumer comme sujet autonome, espace an-archique qui, contre toute forme de subsomption de l'individualité vivante sous la loi d'une quelconque identité, laisse être les différences, libère la diversité dans son éclatement spontané. La finalité pratique de la révolution an-archique oblige alors à ne jamais retomber en-deça de la ligne que trace l'impératif kantien des Fondements de la métaphysique des mours où Kant écrit : " agis de telle sorte que tu traites l'humanité aussi bien dans ta personne que dans la personne de tout autre toujours en même temps comme fin et jamais simplement comme moyen ». La révolution s'oblige à ne plus rabaisser le sujet à un simple moyen, Blobmitte, mais de toujours lui faire droit comme Selbstzweck. Dans l'espace anarchique, plus de principe transcendant, plus d'arché où chaque sujet doit venir s'identifier pour trouver identité, ce modèle de l'identification/individuation qui fait de chaque individu le moyen de sa fin, ce modèle de la normalisation, ce procès de socialisation où l'individu est soumis à un procès dont la finalité lui reste extérieure. Il n'y a plus qu'un système infini de différences où l'individualité est toujours la somme interminable de différences. Chaque sujet trouve son identité non par identification mais par différenciation, il ne s'agit plus de ramener au même, mais de laisser être les différences. La volonté d'unifi-

24. “ $\AA$ une société partielle, parce que fondée sur la poursuite des intérêts d'une classe particulière et sur une conception limitée de l'efficacité, le socialisme oppose la perspective d'une société totale, parce que guidée par l'intérêt de toute la société et sur une conception élargie de l'efficacité . . . Le socialisme de Marx reste pensée comme la coïncidence nécessaire d'une rationalité économique achevée et d'une transparence sociale réalisée " (Rosanvallon, op. cit. p. 146). 
cation disparaît car son principe, l'arché, a été dévalué. Aussi A. Negri peut-il noter que " chaque pas en avant vers le communisme est un moment d'extension et d'expansion de toute la richesse des différences $»$. (p. 291).

Le communisme n'a donc rien de commun avec un mode de production économique ou un mode d'organisation sociale, il est même, au contraire, ce qui définit la règle pratique de leur impossibilité à venir, de l'impraticabilité désormais de leur reconduction. Il n'est pas plus une politique qu'une économie. Ces deux dimensions de l'activité humaine se dissolvent dans le mouvement d'établissement du communisme et ne sauraient donc en définir la logique, une logique qui nous oblige à renverser radicalement la pensée du communisme comme pensée de l'être ensemble, de la communauté des sujets historiques, cette vision qui ordonne le projet révolutionnaire à une téléologie politique, qui lui impose le plitique comme son télos obligé, c'est-à-dire l'organisation de la vie de/dans la cité et qui ressoumet le procès de la révolution aux exigences de la logique identitaire, c'est-à-dire enfin de compte à l'économie-politique du pouvoir. L'espace du politique est un terrain miné, s'y tiennent tous les pièges du pouvoir, et s'y installer c'est se condamner à redévelopper les puissances qui sont au principe d'instauration du pouvoir et de son ordre. D'où la nécessité de s'en retirer, de penser une politique révolutionnaire qui ne soit plus politique, qui ne se donne plus pour fin l'organisation de la cité au nom du supposé bien commun. Il y a derrière la position d'une telle valeur toujours la ruse d'une puissance à l'affût d'une prise de pouvoir. Proudhon a raison : "le gouvernement ne peut être révolutionnaire et même la démocratie la plus radicale comprise comme organisation politique de la cité n'est que l'idée de l'État étendu à l'infini ». (p. 43).

La visée révolutionnaire n'est pas de gouvernement, il s'agit au contraire de rendre impossible tout gouvernement social, ce projet qui a toujours pour fin, sous le couvert de l'universalité affirmée de ses buts, les intérêts d'un groupe particulier. Organiser la cité, c'est toujours dans le même temps organiser un pouvoir (établissement d'un ordre au service d'un groupe). La révolution a pour but de dés-organiser le politique afin de rendre impraticable tout exercice de pouvoir, de décourager toute vél- 
léité de restauration d'une instance de pouvoir dans le rejet radical de toute structuration sociale qui fait primer un principe transcendant sur les individus, un principe d'universalité (ce qui définit en propre toute politique). Laisser valoir la catégorie de l'universel dans la logique de la révolution c'est se condamner à penser la libération de l'individu, l'émancipation révolutionnaire dans les catégories de son oppression. En effet au caractère logique de l'universel, moyen d'une mise en ordre, d'un classement et d'une subsomption sous une loi d'ordonnancement, qui sont les conditions minimales de tout contrôle, correspond son caractère idéologique et politique : catégorie de classement, elle ouvre le champ à un contrôle de ce qui lui a été subsumé, soumission qui est le premier indice d'un pouvoir qui s'installe. Mais à cette fonction s'en ajoute une autre de mystification, l'universel sert de support logique/ontologique à la catégorie politique de l'État, il sert de couverture aux intérêts particuliers qui y sont investis, il est le moyen d'un anonymat dont se recouvre un point de vue particulier. Valeur régulatrice de l'économie dú pouvoir, puissance première de légitimation, sa suppression et sa critique radicale entame le procès de mise à mort du pouvoir et de son ordre. Le principe an-archique dévalue cette valeur de sens en découvrant derrière l'universalité affirmée de l'universel la particularité d'un point de vue, la partialité d'une prise de position, prise de position pour une fiction qui n'est jamais innocente mais sert toujours des intérêts déterminés, ceux d'un pouvoir présent ou à venir. C'est le maintien de cette valeur de sens qui a bloqué, dans l'idéalișme allemand, l'élévation de la nouvelle conception de la subjectivité à sa véritable radicalité. L'universel a la même fonction stabilisatrice que la positivité. Il est dans l'ordre des rapports sociaux ce qu'est la positivité dans l'ordre des rapports au monde. Universalité et positivité sont des qualités qui expriment l'existence d'une transcendance, qui ont toujours une fonction immunisante par rapport à toute mise en question (ce qui énonce une indépendance par rapport au sujet ne saurait être véritablement mis en question par celuici). C'est à ne pas avoir reconnu cette parenté ontologico-politique entre l'universalité et la positivité que l'idéalisme allemand s'est interdit de conduire à son terme sa critique de la métaphysique : il a continué à voir dans l'universel une valeur 
d'émancipation qu'on pouvait opposer à une positivité porteuse de l'aliénation; la loi morale chez Kant, le sollen de Fichte, l'État chez Hegel sont les marques de la soumission de la subjectivité à la norme de l'universalité.

Aussi si la réalisation de l'autonomie est réalisation des conditions d'impossibilité du pouvoir et si tout pouvoir s'autorise toujours d'un universel, c'est toute possibilité de position de l'universel qu'il s'agit d'interdire. C'est pourquoi la pensée de la révolution se laisse définir comme une lutte contre la métaphysique de l'universel.

On trouve une première approximation de la différence entre révolution porté par la volonté politique de fonder un ordre et la révolution an-archique dans une distinction faite par Marx entre révolution politique dont " le point de vue est celui de l'État " et « révolution sociale » qui est "protestation de l'homme contre sa vie inhumaine ". Différence que le marxisme n'a jamais pratiquée préférant s'installer de façon unilatérale et massive du côté des révolutions politiques en développant le projet révolutionnaire sous le schème de l'instauration d'un ordre, de la fondation d'une nouvelle société; visée fondatrice qui ne peut être que la visée d'un sujet dont le point de vue est celui de l'État. Lénine est à nouveau le meilleur exemple de ce modèle de pensée qui se condamne à développer l'aporie révolutionnaire, aporie de vouloir abolir, penser la disparition pouvoir du point de vue même du pouvoir, visée absolument contradictoire s'il est vrai que le seul point de vue possible du pouvoir, le seul qu'il puisse assumer est celui de sa reproduction, toujours élargie, de sa toujours plus grande majoration.

C'est contre un tel détournement des fins révolutionnaires que se définit le communisme comme concept recteur de la pensée an-archique, pensée qui définit quant à elle un individualisme radical qui n'est pas plus retraduction de l'utopie libérale du marché qu'il ne vise la fondation d'une société idéale. Pensée de l'individu, la pensée communiste ne s'inscrit plus dans la problématique des rapports entre société civile et État, du juste équilibre entre ces deux puissances, elle vise au contraire la suppression de la sphère de l'économie comme celle de la politique. Ainsi lorsque Marx dans la Question Juive affirme que 
l'individu " dans l'état politique accompli, mène une vie double, une vie qui se passe dans la communauté politique où il se considère comme un être universel, un être générique, et une vie qui se passe dans la société civile où il travaille comme homme privé » (Werke Tl p. 355), il ne vise pas, en opposition à cette dichotomie, la réalisation de l'universel qu'Hegel n'aurait atteinte que de façon formelle. Par delà la terminologie qui brouille la signification concrète de la visée pratique qui commande tout le mouvement de pensée de Marx lorsqu'il parle d'universalisation de l'homme au niveau de sa vie empirique immédiate, universalisation qu'il lie d'une part à la disparition de l'État, du politique comme sphère séparée, et de l'autre de l'économie comme abolition du travail, cette revendication que la « substance de l'État devienne la vie même des individus " n'aboutit pas à un hyperhégélianisme conduisant à un totalitarisme politique, mais à la destruction sans reste de toute position d'une division interne au sujet. La substance de l'État à réaliser est ici la liberté, l'autonomie réalisée. La revendication de Marx est la revendication d'une réalisation de l'autonomie qui ne s'effectue plus de façon illusoire dans une sphère séparée de la vie concrète et pratique de l'individu, mais dans le mouvement de base de cette vie. Retour de l'autonomie à soi dans la critique destructive de tous les fétiches que construit le monde politique. S'il y donc rien, chez Marx, critique de la séparation et de l'opacité sociale, si les divisions entre société civile et État, entre citoyen et bourgeois occupent bien sa pensée, ce n'est pas au nom de l'établissement d'une communauté idéale, mais au nom de la division qui habite le sujet, de la séparation qui déchire l'individu, de la schizophrénie qui le déchire et le dévore et dont la sphère politique est la mise en scène exemplaire. Aussi la communauté que réalise le communisme n'est pas comme telle sa fin. La communauté n'est pas fin pour elle-même, elle ne peut plus définir l'objet qui normalise le mouvement de la révolution et lui assigne ses finalités positives, elle n'est plus que résultat. L'être-ensemble des individus ne se pose plus par rapport aux individus comme un apriori, une finalité donnant à son mouvement sa loi. Il est résultat a posteriori de la multiplicité des mouvements autonomes de chacun des sujets ${ }^{25}$. Il

25. «Tout repose sur l'individu remarque G. Haascher au terme de son livre dans la société communiste : c'est lui qui institue, spontanément et à partir de soi, de sa nature, de son 
n'est plus l'a-priori qui fixe l'ordre des finalités de chaque pratique individuelle (renvoyant alors l'individu à la forme d'individu moyen d'une fin le transcendant et faisant retomber la révolution dans le domaine de l'hétéronomie), mais effet aposteriori de l'infinité des libertés monadiques qui n'ont plus pour fin que le maintien de leur libre mouvement ${ }^{26}$. Ainsi la "production " révolutionnaire du nouveau sujet vise une sujectivitélindividualité qui ne ménage plus dans le sujet de division où le pouvoir puisse trouver possibilité d'exercer sa puissance, vise à détruire dans le sujet toute surface possible d'inscription du pouvoir. Il ne s'agira donc pas pour la révolution de construire un espace transparent, de produire une transparence de la société à elle-même mais de rendre opaque l'espace social au point qu'aucun pouvoir ne puisse plus avoir de prise, que toute volonté d'instauration d'un pouvoir soit réduite à l'échec, à une tentative absurde de maîtrise de ce qui ne se laisse plus soumettre à l'hétéronomie mais se définit toujours comme la fin suprême et a pour finalité la reproduction de son existence comme autonomie.

Processus de destructuration sans fin, de différenciation toujours continuée, règne de la différence réalisée contre le règne de l'identité, de la multiple irréductible contre celui de l'unité accomplie, règne de l'ouverture jamais clôturable qui renvoie alors l'angoisse de la dispersion à son vrai lieu d'origine, non pas la société qui serait menacée d'entrepie, mais celui du pouvoir pour qui la dispersion est signe de son impuissance, équivaut à sa perte de réalité et est son principe de mort.

\section{Maison des Sciences de l'Homme}

Paris

fonds le plus intime, le lien social, sans que celui-ci doive être assigné à une transcendance quelconque : l'individu est l'universel, aucune altérité ne le commande du dehors, ne l'extériorise ou ne le met à distance de soi, le forçant par là même à se chercher aux confins de son monde . . . Sublimation radicale de l'individu, devenu par la même proprement infini " (op. cit. p. 290). Contrairement à la thèse de Haarscher pour qui une telle perspective ne peut être tracée qu'au prix d'une décorporéisation totale de l'individu, nous y voyons le mouvement d'une réappropriation possible de son corps perdu, aliéné dans le mouvement d'une activité qui signifie, à travers la vente de la force de travail et sa contrainte, sa dépossession, son aliénation au pouvoir étranger d'un autre qui se donne le droit de déterminer sa liberté.

26. Excerpts fron James MILL's Elements of political Economy Early Writtings Marx The Pelican Marx's Library, p. 265. 


\section{BIBLIOGRAPHIE}

M. RUBEL, Marx critique du marxisme. Payot, 1976.

G.W.F. HEGEL, La science de la logique. Trad. de V. Jankelevitch, Aubier.

J.L. PETIT, Du travail vivant au système des actions, Seuil, 1980.

J. DERRIDA, L'écriture et la différence. Seuil.

J. DERRIDA, Marges de la philosophie. Éditions de Minuit.

R. SCHÜRMANN, Le principe d'anarchie. Seuil, 1982.

A. NEGRI, Marx au delà de Marx. C. Bourgeois, 1979.

M. HENRY, Marx. Gallimard, 1974.

T. MEYER, Zwiespalt . . . Scriptor Verlag, 1973.

A. FRÄNTZKI, Der misverstandenee Marx. Verlag O. Nesde 1980.

M. LANGE, Das Arbeitsprinzip. VERLAG Ullstein, 1980.

G. LeBrun, Kant ou la fin de la métaphysique. A. Colin, Paris 1970.

G. LEBRUN, La patience du concept. Gallimard, 1972.

A. JAULIN, La peau du marxisme. Éditions TER, 1979.

J.L. NANCY, Rejouer le politique. Éditions Galilée, 1980.

GORZ, Adieu au prolétariat, Seuil, 1980.

M. RAYAN, Marxism and deconstruction. The Johns Hopkins University Press, 1982.

ROSANVAllon, L'âge de l'autogestion. Seuil.

ROSANVALlon, L'âge de l'autogestion. Seuil, 1976.

G. HAARSCHER, L'ontologie de Marx. Éditions de l'Université de Bruxelles.

M. RUBEL, Marx critique du marxisme. Payot, 1976.

M. RUBel, Pages de Karl Marx. Payot. 